\title{
Incorporating High Value Care into Undergraduate Medical Education
}

\author{
Erik Faber ${ }^{*}$, Daniel Wells \\ Medical School, University of Minnesota, USA
}

Copyright $(2017$ by authors, all rights reserved. Authors agree that this article remains permanently open access under the terms of the Creative Commons Attribution License 4.0 International License

\begin{abstract}
Identified Need: The United States spends the most per capita on healthcare, but ranks much lower than most industrialized nations in quality and many health metrics. Studies have shown higher costs do not translate to high quality care, and may indeed translate to lower quality care and patient experience. Teaching high value care (HVC) is only formally integrated into $15 \%$ of internal medicine residency programs and hardly into undergraduate medical education. Innovation: To address rising healthcare expenditures in the United States, the innovation trains physicians starting in year one of undergraduate medical education in a HVC focused curriculum regarding diagnostic testing. To create the curriculum, content will be introduced throughout each year of training. Each consecutive year, the fundamental principles will be reinforced with the goal to have students practicing HVC at the end of their clinical training. Implications: This training could help decrease unnecessary test ordering common among residents and practicing physicians. Our health system will benefit through the cost savings associated with the decrease in unnecessary medical testing. Patients will also benefit because only the most valuable tests will be ordered, saving the patient unnecessary distress, time, and exposure to adverse events.
\end{abstract}

Keywords High Value Care, Undergraduate Medical Education, Curriculum, Health Care Costs, Diagnostic Techniques and Procedures, Medical Students, Cost Conscious Care, Test Ordering

\section{Background}

The United States has a serious issue with healthcare expenditures, spending the most per capita on healthcare but ranking much lower than most industrialized nations in quality and many health metrics.[1] This problem is addressed as a main tenet of the quadruple aim, which seeks to improve healthcare by improving population health, physician well-being, the patient experience, and reducing healthcare expenditures. Many studies have shown higher costs do not translate to higher quality care, and may indeed translate to lower quality care and patient experience.[2-3] Furthermore, a number of studies have demonstrated an association between the setting in which physicians train and their future clinical decisions in terms of cost. $[2,4]$ Clearly, drastic institutional and educational changes must take place to combat this costly issue. One way to address this issue is to focus on undergraduate medical education (UME) as a way to train future physicians to provide high value care (HVC), a concept aimed to provide excellent patient care while simultaneously reducing healthcare costs. The main purpose of UME is to train students for residency and future practice in the constantly evolving world of medicine. In particular, it has been observed that residents currently order more tests than experienced doctors, some of which may not be necessary.[5-7] It has been estimated that of the $\$ 765$ billion in healthcare waste, about $\$ 210$ billion (or $27.5 \%$ ) is due to unnecessary services that includes redundant testing.[8] In both UME and graduate medical education (GME), instructions of when and how to apply diagnostic tests has not kept pace with the rise of imaging and development of medical technology. Many practicing physicians and residents are unaware of the costs of the tests they order on a daily basis.[9-10] In a recent survey, only 1 in 4 internal medicine residents knew where to find these costs and share them with patients, and less than half of these residents incorporated cost into their decision making.[11] By making providers aware of the cost, the cost of services can be significantly reduced without sacrificing quality of care.[12] We believe some of this excess testing could be curbed by better preparation throughout UME.

Currently, the majority of medical students are not exposed to a formal curriculum on the selection of diagnostic testing and its implications for HVC, especially during the first two years of training. Traditionally, this training is embedded in the medical clerkships where students are exposed to practical day-to-day patient care in a variety of clinical specialties. However, by design, this 
training is not comprehensive. Curriculum for the individual student depends heavily on the cases seen during a short rotation for students and the acumen of their faculty to teach HVC. Next, in GME, exposure to HVC is minimal and inconsistent between residency programs. In a study compiled by the Association of Program Directors for Internal Medicine, only $15 \%$ of directors reported their programs offer formal curriculum in "cost-conscious care," while $48 \%$ of programs believed their faculty model cost-conscious care.[13] Considering the data, we find GME training insufficient to produce future physicians who practice HVC. If we allow future physicians to be trained to mimic all the habits of current faculty, we are unlikely to curb the high cost of healthcare attributed to unnecessary testing.

In a recent review of teaching HVC in medical school, three components were identified as essential for success: effective knowledge transmission, reflective practice, and a supportive environment.[14] Our proposal seeks to begin addressing the first component during the first year of medical school, the second beginning the clerkship years, and the third throughout. If we were to create a medical school of the future, we would want it to prioritize curriculum that emphasizes $\mathrm{HVC}$ as a way to reduce costs and improve the patient experience. The curriculum will train more value-focused students to go on to clinical clerkships, residency, and professional practice using resources more effectively.

\section{High Value Care Curriculum}

To create our HVC curriculum, we will introduce content throughout each year of training, furthering the fundamental principles each consecutive year with our goal to have students practicing $\mathrm{HVC}$ at the end of their clinical training. During the first year, we will begin to formally introduce the diagnostic tests available to physicians. Although the detailed mechanism of the test may not be necessary for a physician to know, the information gleaned from the test is important. Even more crucial may be what is not confidently known. Future physicians need to be aware of the dearth of data on efficacy of many tests if they are to practice HVC and improve on current technologies. We would also introduce students to what patients experience during testing. For example, we can include patient narratives of what it is like to have an MRI and have students participate in an oral glucose tolerance test. This instruction will help students develop empathy for their patients and complements the more data-driven instruction behind HVC. Another important component would be for students to compose a mock bill for medical expenses in a small group setting, leading to a discussion on potential ways to reduce cost. Along with a basic science background, we believe it is necessary for first-year medical students to learn the basics of diagnostic testing concurrently with interviewing and physical exam skills.

Typically, the next segment of medical school is an introduction to pathophysiology. During this time, we will expose students to the available and preferred tests for the conditions they are learning about and the reason behind test ordering. They will also learn the sensitivity and specificity of each test for the diseases they study, as well as the concept of pretest probability affecting the positive and negative predictive value of a given test. These components of test performance are an important component of the delivery of HVC. Our plan is that through association of the most appropriate test with the disease, and learning which tests not to use, students will more confidently and appropriately order tests throughout their clinical years. It is as imperative to know how each tool in your medical toolkit works as it is to know what tools are available.

During the clinical clerkship years, this HVC training will be applied and further developed across most, if not all, specialties ideally. Some medical schools have experimented with the role of the medical student as part of the care team; the undergraduate medical student often has more time with each patient than other members of the care team. Some schools have begun training students to write SOAP-V notes on their patients, where the "V" stands for "value."[15] In these notes, the student comments on the effectiveness of the plan as it pertains to cost of the plan, effectiveness of the plan, and also possible better viable alternatives (suggested by the literature or otherwise). In this way, the student is more an active participant in review of the patient care the team delivers. Not only are these students in the mindset of HVC, but they are modeling the habits of HVC delivery to the other members of the team who are receiving immediate feedback on their actions. Their voice is undeniably valuable as they become the teachers, shifting the paradigm of unnecessary test ordering. In a recent small study, $86 \%$ of third-year medical students in their internal medicine rotation were able to identify unnecessary tests ordered and noted a major factor for this waste as "ingrained practices."'[16] An audit and feedback of the team's plan, along with a time for reflection on these practices, seems to be integral in learning HVC.[14] We believe this clinical experience would be the culminating training experience of undergraduate medical students to then enter residency with more confidence in their diagnostic test ordering ability.

\section{Resources}

One attractive aspect of a curriculum centered around HVC is the limited resources required to implement this plan. We believe this training could either be part of a reformed basic science curriculum, replacing less relevant topics that currently are taught and be part of the introduction to critical reading of the medical literature. We do not foresee this training and exposure to consume much 
instructional time for students. It would require time and compensation for faculty members to design the introductory course for first year medical students to learn current diagnostic techniques. Resources available to instructors include but are not limited to Cochrane reviews and meta-analyses. In addition, the American College of Physicians has created a 6-hour HVC curriculum intended to be adapted for UME. For the incorporation of appropriate tests into pathophysiology, we believe course instructors will be able to add the available information on HVC to their content without substantial additional time and effort. The SOAP-V note training program is already being implemented by a number of medical schools, so the infrastructure exists to adopt this model to medical schools more broadly. Because many residency programs have started to incorporate HVC into their curriculum, a partnership between UME and GME curricula could be beneficial to provide medical students with practical examples of HVC.[17] Ultimately to create a successful pilot program, the enthusiastic involvement of faculty, staff and students is crucial to make a curriculum that will meet the goals we set forward. We believe these small but significant changes to the UME curriculum will produce residents and future physicians who are increasingly savvy about diagnostic test ordering, and who see the tools available as resources whose benefit must be carefully considered prior to their use.

\section{Implementation}

Initially, we aim to pilot our comprehensive HVC curriculum at a few institutions and gain support of the training sites where medical students rotate. In this pilot, we will track students exposed and unexposed to this HVC curriculum into residency to observe any difference in confidence in test ordering and in number and cost of tests ordered. In addition, measuring patient satisfaction, care appropriateness, and health outcomes for those cared for by residents trained in HVC will ensure this training is ultimately benefiting patient experience. This observational study will provide quantitative and qualitative outcomes of our training in healthcare cost reduction and patient experience improvements. Results of the study can be used in support of implementing this curriculum on a mass-scale. Support from the American Association of Medical Colleges and American Medical Association would be essential in adapting this innovation to all U.S. medical schools, as these institutions are key players in UME. Perhaps an inclusion of HVC in USMLE Step 1 or 2 would quickly prioritize this element for instruction on a national scale, as it has done for certain residencies such as internal medicine whose culminating exam now tests this concept. This new curriculum will be a significant step in creating a generation of physicians dedicated to improving the value of healthcare. Medical students will benefit from the introduction of more clinical content earlier in their education. Patients will benefit because only the most valuable tests will be ordered, saving the patient unnecessary distress, time, and exposure to adverse events. Our health system will benefit through the cost savings associated with the decrease in unnecessary medical testing. A nation-wide implementation of this curriculum will ensure all medical students are exposed to high value care training that could decrease regional differences in health care expenditures that add to rising costs. Ultimately, we aim to accomplish the quadruple aim with improved patient care experience and decreased cost of care delivery. While test ordering is one component of excess cost, increasing the practice of HVC has the potential to save the entire healthcare system money. Money not spent on healthcare is money spent elsewhere. In this way, we all benefit from smarter care.

\section{REFERENCES}

[1] Berwick DM, Nolan TW, Whittington J. The Triple Aim: Care, Health, and Cost. Health Aff (Millwood). 2008 May/June; 27(3): 759-69.

[2] Fisher E, Goodman D, Skinner J, Bronner K. Health Care Spending, Quality and Outcomes: More Isn't Always Better [Internet]. The Dartmouth Institute for Health Policy and Clinical Practice; 2009 [cited 2015 Dec 5]. Available from: http://www.dartmouthatlas.org/downloads/reports/Spending Brief_022709.pdf

[3] Fisher ES, Wennberg DE, Stukel TA, Gottlieb DJ, Lucas FL, Pinder EL. The implications of regional variations in Medicare spending. Part 1: the content, quality, and accessibility of care. Ann Intern Med. 2003 Feb 18; 138(4): 273-87.

[4] Chen C, Petterson S, Phillips R, Bazemore A, Mullan F. Spending patterns in region of residency training and subsequent expenditures for care provided by practicing physicians for Medicare beneficiaries. JAMA. 2014 Dec 10; 312(22): 2385-93.

[5] Chandra A, Khullar D, Wilensky GR. The economics of graduate medical education. N Engl J Med. 2014 Jun 19; 370(25): 2357-60.

[6] Fung D, Schabort I, MacLean CA, Asrar FM, Khory A, Vandermeer B, et al. Test ordering for preventive health care among family medicine residents. Can Fam Physician. 2015 Mar 1; 61(3): 256-62.

[7] Wynn BO, Smalley R, Kristina M. Cordasco. Does it cost more to train residents or to replace them? A look at the costs and benefits of operating graduate medical education programs [Internet]. RAND Corporation; 2013 [cited 2015 Nov 17]. Available from:

http://www.rand.org/pubs/research_reports/RR324.html

[8] The Healthcare Imperative: Lowering Costs and Improving Outcomes: Workshop Series Summary [Internet]. Washington, D.C.: National Academies Press; 2010 Dec 
[cited 2017 May 9]. Available from: http: //www.nap.edu/catalog/12750

[9] Carlson J, Dachs RJ. Family medicine residents remain unaware of hospital charges for diagnostic testing. Fam Med. 2015 Jun; 47(6): 466-9.

[10] Tek Sehgal R, Gorman P. Internal medicine physicians' knowledge of health care charges. J Grad Med Educ. 2011 Jun; 3(2): 182-7.

[11] Ryskina KL, Smith CD, Weissman A, Post J, Dine CJ Bollmann K, et al. U.S. Internal Medicine Residents' Knowledge and Practice of High-Value Care: A National Survey. Acad Med. 2015 Oct; 90(10): 1373-9.

[12] Silvestri MT, Bongiovanni TR, Glover JG, Gross CP. Impact of price display on provider ordering: A systematic review. J Hosp Med. 2016 Jan; 11(1): 65-76.

[13] Patel MS, Reed DA, Smith C, Arora VM. Role-Modeling Cost-Conscious Care--A National Evaluation of Perceptions of Faculty at Teaching Hospitals in the United States. J Gen Intern Med. 2015 Sep; 30(9): 1294-8.

[14] Stammen LA, Stalmeijer RE, Paternotte E, Oudkerk Pool A, Driessen EW, Scheele F, et al. Training Physicians to Provide High-Value, Cost-Conscious Care: A Systematic Review. JAMA. 2015 Dec 8; 314(22): 2384-400.

[15] Moser EM, Huang GC, Packer CD, Glod S, Smith CD, Alguire PC, et al. SOAP-V: Introducing a method to empower medical students to be change agents in bending the cost curve. J Hosp Med. 2016 Mar; 11(3): 217-20.

[16] Tartaglia KM, Kman N, Ledford C. Medical Student Perceptions of Cost-Conscious Care in an Internal Medicine Clerkship: A Thematic Analysis. J Gen Intern Med. 2015 Oct; 30(10): 1491-6.

[17] Bowman J, Duran A, Duffy B, Gladding S, Baum K. Teaching high-value care: a novel morning report. Clin Teach. 2015 Jun; 12(3): 165-70. 